\title{
Familia, género y sexualidad en las teleseries chilenas contemporáneas. Afinidades con la teoría sociológica y los estudios realizados en el país
}

\author{
Francisco Vidal Velis \\ Universidad Metropolitana de Ciencias de la Educación, Santiago, Chile \\ Email: vidalvelis@yahoo.com
}

\begin{abstract}
Resumen: Los temas de familia, género y sexualidad han sido abordados por distintos autores e investigadores, quienes sostienen la producción de importantes cambios en estos ámbitos. El presente artículo describe las formas familiares, las relaciones de género y la sexualidad que difunden cinco teleseries chilenas, resultados que se discuten a la luz de la teoría sociológica y de los estudios realizados en el país. Se trata de un estudio interpretativo basado en principios de la etnografía, del bricoleur y de la grounded theory. De los resultados llama la atención la importancia que adquieren las familias reconstituidas -escasamente abordadas en los estudios nacionales-, los modelos alternativos de género y la diversidad de relacionamientos sexuales contenidos en estos programas, aspectos que la sociología considera característicos de la sociedad moderna. Se plantea la necesidad de ampliar las categorías sociológicas usadas en los estudios, incorporando nuevos tipos de familia, particularmente las reconstituidas y homoparentales.
\end{abstract}

Palabras clave: Familias; comportamiento sexual; relaciones de género; teleseries; sociología

Family, gender and sexuality in contemporary chilean soap operas. Affinities with the sociological theory and the studies carried out in the country

\begin{abstract}
The themes of family, gender and sexuality have been addressed by different authors and researchers, who support the production of important changes in these areas. This article describes the family forms, the gender relationships and sexuality that are broadcast by five Chilean soap operas, results that are discussed in light of the sociological theory and the studies carried out in the country. It is an interpretative study based on principles of ethnography, bricoleur and grounded theory. Of the results, attention is drawn to the importance acquired by reconstituted families -subsequently addressed in national studies-, the alternative gender models and the diversity of sexual relationships contained in these programs, aspects that sociology considers characteristic of modern society. There is a need to broaden the sociological categories used in the studies, incorporating new types of families, particularly reconstituted and homoparental ones.
\end{abstract}

Key words: Families; sexual behavior; gender relations; soap operas; sociology

\section{Família, gênero e sexualidade nas séries contemporâneas chilenas. Afinidades com a teoria sociológica e os estudos realizados no país}

Resumo: Os temas família, gênero e sexualidade têm sido abordados por diferentes autores e pesquisadores, que apoiam a produção de importantes mudanças nessas áreas. Este artigo descreve formas de família, relações de gênero e sexualidade disseminadas por cinco séries televisivas chilenas, resultados que são discutidos à luz da teoria sociológica e dos estudos realizados no país. Trata-se de um estudo interpretativo baseado nos princípios da etnografia, do bricoleur e da teoria fundamentada. Destes resultados, chama-se a atenção para a importância adquirida pelas famílias reconstituídas subseqüentemente abordadas em estudos nacionais -, os modelos alternativos de gênero e a diversidade de relações sexuais contidas nesses programas, aspectos que a sociologia considera característica da sociedade moderna. Existe a necessidade de ampliar as categorias sociológicas utilizadas nos estudos, incorporando novos tipos de famílias, particularmente 
reconstituídas e homoparentais.

Palavras-chave: Famílias; comportamento sexual; relações de gênero; séries de TV; sociologia

$* * *$

\section{Introducción. Cambios sociales, cambios familiares}

En esta introducción se analizan y discuten algunos cambios que parece experimentar la familia chilena en las décadas recientes, así como la forma en que ésta se ha visto impactada por otras transformaciones ocurridas en la sociedad, como las devenidas del género y la sexualidad. Para enriquecer el análisis, estos cambios se analizarán considerando los planteamientos de la teoría sociológica contemporánea y los resultados de los estudios realizados en el país. Estos últimos serán tratados someramente en este punto, ya que se profundizarán en el marco teórico, donde, además sus resultados serán contrastados con los obtenidos décadas atrás. Creemos que la incorporación del punto de vista sociológico e investigativo nos permitirá comprender de mejor manera la naturaleza y el carácter de las transformaciones que vive la familia en la actualidad. En su parte final, el apartado incluye una fundamentación de las razones por las que optamos por analizar la familia, el género y la sexualidad a partir de su representación televisiva.

Uno cambio importante tiene que ver con la conceptualización de familia, ya que, en la actualidad, la discusión se ha complejizado bastante, criticándose incluso la tenencia de hijos/as como elemento diferenciador (del Picó Rubio, 2011), ya que no todas las parejas quieren o pueden tener hijos, como las parejas infértiles o las homoparentales. También está el caso de la maternidad subrogada (Rodríguez y Martínez, 2012) y el de las madres solteras por elección, “aquellas que, de un modo voluntario y consciente, sin formar parte de un núcleo matrimonial o de pareja, han decidido tener hijos en solitario, bien mediante técnicas reproductivas, bien mediante adopciones” (Moncó, Jociles y Rivas, 2011, p. 73). Por otra parte, la importante ola migratoria que vive el país ha puesto el acento en las familias transnacionales, concepto que, para Gonzálvez (2016), debe entenderse a partir del parentesco en su relación con el género. En el caso de nuestro país, las investigaciones muestran que la familia nuclear tradicional se ha venido debilitando desde los años 70, fenómeno que se habría profundizado en las décadas siguientes, donde

“esta configuración familiar ha perdido relevancia y peso en relación al conjunto de los núcleos familiares (..), ya no está en el centro de las políticas públicas ni tampoco en la de los actores que tienen la capacidad de decidir sobre ésta” (Olavarría, 2014, p. 491).

Por otro lado, muchas personas que se divorcian no lo hacen para quedarse solteras, sino para formar nuevas parejas, formalizadas o no, convirtiéndose en una nueva familia, la que podría tener características distintas de las tradicionales ${ }^{1}$, las familias reconstituidas ${ }^{2}$. En general, sabemos poco de estas familias, ya que, en nuestro país, los instrumentos de recolección de información no la incorporan como categoría, subsumiéndola dentro de la familia nuclear, en el entendido que hay padre, madre e hijos, aun cuando no sepamos si todos los hijos fueron engendrados por quienes viven con ellos (Ministerio de Desarrollo Social, 2015, 2017). En contraposición al ideal de permanencia del objeto amoroso del amor romántico, las familias contemporáneas, particularmente las reconstituidas, se ven afectadas por el carácter transitorio de los lazos afectivos que los unen, cambio que ha sido problematizado desde la teoría sociológica. Bauman con su metáfora de la modernidad líquida, donde esta temporalidad se refleja en el concepto de "relaciones de bolsillo", que "pueden sacarse en caso de necesidad, pero también pueden volver a sepultarse en las profundidades del bolsillo cuando ya no son necesarias” (2007, p. 10).

Beck hace lo propio con su idea de "la familia negociada a plazo"3 ; Giddens (2008) con la del "amor confluente” y Eva Illouz (2009) con la del “amorío”. Así, uno de los cambios más relevantes de la modernidad, tendría que ver con el ámbito de la intimidad: la familia, el género y la sexualidad. Al respecto, Giddens estima que:

“ninguno (de los cambios) supera en importancia a los que tienen lugar en nuestra vida privada -en la sexualidad, las relaciones, el matrimonio y la familia. Hay en marcha una revolución mundial 
sobre cómo nos concebimos a nosotros mismos y cómo formamos lazos y relaciones con los demás” (2003, p. 65).

La incorporación de la mujer al mundo laboral ha devenido en cambios en las relaciones entre los géneros, fenómeno que Giddens denomina "relaciones puras", y que entiende como "una relación de igualdad sexual y emocional que tiene connotaciones explosivas respecto de las formas preexistentes de poder entre los diversos pareles sexuales establecidos" (2008, p. 11-12). El autor también apunta al inédito proceso de "negociación sexual” que deben enfrentar las parejas de hoy, que deberán llegar a acuerdos sobre qué hacer y no hacer en la relación sexual, democratizándose el acceso al placer para ambos géneros y poniendo al hombre la tarea de satisfacer sexualmente a su pareja, algo muy distinto a lo que acontecía con anterioridad (Sharim, Silva, Rodó y Rivera, 1996). Así, las familias no sólo habrían cambiado en términos sociodemográficos ${ }^{4}$, sino también por las transformaciones en las relaciones de género y en las formas de vivenciar la sexualidad que predominan en la sociedad en un momento determinado.

En tal sentido, una investigación mostró que, para los/as participantes, "las transformaciones culturales -tendientes a la igualdad y el declive de los referentes tradicionales de género- han impactado en sus relaciones de pareja” (Sharim y Rihm, 2017, p. 7). Por otro lado, las familias se hacen más democráticas y sus relaciones se vuelven más horizontales, en contraposición a la familia industrial, donde la autoridad recaía de manera exclusiva en el padre (Giddens, 2010; Olavarría, 2011).

A su vez, las familias deben abordar de modo más directo el tema de la sexualidad con los hijos/as, considerando el adelanto en el inicio sexual y la necesidad de discutir sobre métodos preventivos e infecciones de transmisión sexual. Incluso, algunos padres y madres permiten que sus hijos/as tengan relaciones sexuales dentro de la casa familiar, situación que habría causado escándalo años atrás. Por otro lado, los hijos/as asumen que sus padres tienen vida sexual activa, a diferencia de lo que ocurría en tiempos pretéritos, donde la sexualidad de los padres se mantenía a resguardo de la mirada de los hijos/as. Desde el punto de vista de los hombres, las investigaciones muestran que uno de los principales cambios en la familia es "la consolidación de una relación afectiva más horizontal con los hijos” (Araujo y Martuccelli, 2012, p. 161). En igual sentido, el mayor involucramiento de los hombres en la crianza "se evidencia también en una relación más afectuosa con los hijos” (Saldaña, 2018, p. 197). Así, en las sociedades contemporáneas:

"Se ha puesto en jaque la teoría de los roles sexuales y se ha iniciado un proceso de desideologización de las relaciones entre hombres y mujeres, tanto en sus identidades como en las relaciones de género con sus parejas e hijos” (Olavarría, 2001, p. 30).

El presente artículo analiza los cambios que ha experimentado la familia, el género y la sexualidad en las décadas recientes, análisis que se realiza a partir de la forma en que éstas son representadas en teleseries chilenas contemporáneas. En particular, se abordarán los tipos de familias que difunden estos programas, la relación que mantienen las parejas entre sí, el trato con los hijos/as y el modo en que se vivencia y "se negocia" la sexualidad. Así, el objeto de estudio son las transformaciones familiares, sexuales y de género, las que son descritas y analizadas a partir de su representación en las teleseries, que, en este caso, vendrían a ser las unidades de análisis. El interés de estudiar esta representación radica en las relaciones de intercambio que se pueden observar entre la familia representada y la familia real, ya que, como fundamentamos en la metodología, sus directores y guionistas crean los personajes tratando que el televidente se vea reflejado en ellos, tratan conscientemente de emular la realidad, efecto que se logra con focus groups, que miden su efecto en la opinión pública y con la contratación de 'oidores', personas que van a pubs y bares a escuchar conversaciones ajenas, que podrían ser útiles en la creación de los personajes (Santa Cruz, 2003).

Por otro lado, al incorporar problemáticas sociales controvertidas, como las relaciones familiares o de género, las teleseries ponen en discusión temas sobre los cuales existen distintas posturas en la sociedad, incluso, en ocasiones, anticipando temas, tal como habría hecho, en 2007, Alguien te mira con los femicidios (Vásquez, 2016). Recordemos también el debate por la homosexualidad del personaje de Ariel Mercader en "Machos" (2003), personaje que contrasta con las estereotipadas y caricaturescas representaciones anteriores, como la de Amadeo en “Trampas y caretas” (1992) y de Pierre Lefont en “Marrón glacé” (1993). 
El siguiente apartado describe los resultados de algunas investigaciones recientes sobre familia, género y sexualidad realizadas en Chile, para, acto seguido, contrastarlos con los obtenidos en estudios de un par de décadas atrás. Esta contraposición no sólo nos permitirá aprecian mejor los cambios producidos en estos ámbitos, sino también como elementos de juicio para analizar la representación que hacen las teleseries.

\section{Marco teórico}

En los 80 y 90, la familia se definía en función del matrimonio y la tenencia de hijos/as (Morandé, 1994). Sin embargo, con la aprobación de la ley de divorcio, no parecía factible seguir sosteniendo que el matrimonio fuese lo distintivo de la familia, manteniéndose sólo a los hijos como elemento diferenciador (del Picó Rubio, 2011). En la actualidad, la discusión científica parece cuestionar tanto la concepción tradicional de familia como la de parentesco (Konvalinka, 2010; Stone, 2007), estimándose que "ha dejado de estar claro quién o qué constituye una familia. Los límites se hacen borrosos, las definiciones vacilantes; crece la inseguridad” (BeckGernsheim, 2003, p. 13). A su vez, Parkin y Stone observan "una tensión entre el parentesco como elección y construcción social y la concepción cultural más antigua del parentesco en tanto enraizado en la reproducción biológica” (2007, p. 547). En un contexto en que las parejas duran cada vez menos tiempo juntas y que la sangre pierde relevancia como elemento definitorio del parentesco, parece sensato flexibilizar los modos de conceptualizar la familia, definiéndola como una:

"unión de personas que comparten un proyecto vital de existencia en común, que se quiere duradero, en el que se generan fuertes sentimientos de pertenencia a dicho grupo, existe un compromiso personal entre sus miembros y se establecen intensas relaciones de intimidad, reciprocidad y dependencia" (Rodrigo y Palacios, 1998, p. 33) .

Los estudios de familia reportan una transformación importante en las actitudes de los chilenos/as hacia la familia y en los tipos de familia que predominan en el país. Sobre el primer punto, el estudio de Herrera y Teitelboim (2010) muestra una mayor apertura hacia las formas familiares emergentes y la legitimación de nuevos tipos de relacionamientos entre las personas y con sus hijos/as. El 73,8\% de los consultados/as estuvo de acuerdo con que las parejas convivan antes de casarse y el 77,1\% piensa que el divorcio es la mejor solución cuando la pareja no puede resolver sus problemas. Se apoya la crianza de madres y padres solteros y, en temas de reproducción, hay un fuerte apoyo a la píldora del día después y a las técnicas de reproducción asistida. Los estudios de la Universidad Católica y Adimark indican que, desde 2006, todas las afirmaciones tradicionales sobre familia han ido perdiendo apoyo entre los ciudadanos/as. El acuerdo con la afirmación "El matrimonio es un compromiso para toda la vida" desciende del 77\% al 52\% entre 2006 y 2016. Lo mismo ocurre con "Hay que pensar en casarse cuando vienen los hijos," que lo hace del 54\% al 31\%; mientras que "Seguir juntos por el bien de los hijos, aunque la pareja no se lleve bien”, hace lo propio desde el $29 \%$ al $20 \%$ en igual período. La única afirmación que incrementa su aprobación es el matrimonio homosexual, que pasa del 32\%, en 2006, al 54\% diez años después (Universidad Católica y Adimark, 2006; 2016).

Los datos del Registro Civil (2016) muestran una disminución progresiva de los matrimonios durante los últimos años, con una estabilización en 2011, y un aumento de los divorcios entre 2005 y 2009, que también se estabiliza a partir de esa fecha. Las cifras de convivencia se triplican en el transcurso de 20 años. En 1990, el $3,7 \%$ de los hombres y el 3,5\% de las mujeres convivía, mientras que, en 2011, los hombres aumentan al 11,6\% y las mujeres al 10,6\% (Ministerio de Planificación, 2011). Esta tendencia se hace más marcada en la población joven, donde un 16\% de las parejas convive (Instituto Nacional de la Juventud, 2012). Los hogares con jefatura femenina alcanzan la inédita cifra del 42,7\% del total (Ministerio de Desarrollo Social, 2017). También hay cambios en el estado civil del jefe/a de hogar de las familias nucleares, ya que, entre 1990 y 2009, los casados disminuyen del $64 \%$ al 45,7\%; mientras que todas las otras categorías aumentan: las convivencias del 6,1\% al $15 \%$; los/as solteros/as desde el $11,9 \%$ al 18,2\% y la categoría anulado/a, separado/a o divorciado/a lo hace del 11,9\% al 18,2\% (Ministerio de Planificación, 2009). La proporción entre familias biparentales y monoparentales también sufre alteraciones:

“En 1990 el 55,7\% de los hogares eran familias nucleares biparentales, y un 8,5\% familias nucleares 
monoparentales. En tanto, en 2015 la proporción de familias nucleares biparentales se redujo a un 44,7\% y las familias nucleares monoparentales aumentaron a un 14,5\% del total de hogares” (Ministerio de Desarrollo Social, 2015, p. 3).

Por otro lado, los estudios sobre sexualidad muestran un adelanto en la edad de iniciación sexual, mayor diversificación en los repertorios sexuales de las personas y, en términos de sexualidad femenina, un aumento del número de parejas sexuales, mayor declaración de acceso al placer y una incipiente separación entre afectividad y sexualidad, que se expresa en encuentros sexuales en contextos de ocasionalidad. Sobre iniciación sexual, un estudio mostró que los hombres de todas las edades se iniciaban más tempranamente que las mujeres, sin evidenciarse un descenso en las cohortes más jóvenes, como si se aprecia en el caso de las mujeres, que sí muestran cambios en sus edades de iniciación (Mella, Oyanedel, Vargas y de Ugarte, 2015). Los estudios realizados sólo con jóvenes concluyen que las edades de iniciación entre hombres y mujeres son muy similares, los primeros se iniciarían a los 16.36 años, mientras que las segundas a los 16.84 (Instituto Nacional de la Juventud, 2015, p. 98). Sobre parejas sexuales en el último año, el mismo estudio arrojó que, en promedio, las personas reportaban 1.99 parejas; 2.06 los hombres y 1.52 mujeres. En población general, el estudio de Adimark (2017) encontró mayores diferencias, con 8.3 parejas en hombres y 3.2 en mujeres (ADIMARK; 2017). Así, las nuevas generaciones parecieran tener un comportamiento sexual distinto al de la población general, incluso considerando al mismo grupo, comparados por tramos de edad ${ }^{6}$. Este cambio en la vida sexual de la mujer contrasta con estudios anteriores, donde las mujeres de mayor edad reportaban sólo una pareja sexual en la vida, en un $61,2 \%$ de los casos y había mayor diferencia en la edad de iniciación respecto de los varones (Ministerio de Salud, 2000; Valdés, Benavente y Gysling, 1999).

En cuanto a repertorio sexual, el estudio en jóvenes indica que el 49\% de ellos declaró practicar sexo oral, cuatro puntos más que en la medición del 2012, con una distribución por sexo de 52\% para hombres y 46\% para mujeres. En 2000, sólo el 36\% de las mujeres y el 37\% de los hombres realizaba esta práctica (Ministerio de Salud, 2000). Además, el 26\% realiza sexo anal, 31\% de hombres y 21\% de mujeres. No obstante, esta práctica aumenta con la edad, llegando al 35\% en el tramo de 25 a 29 años (Instituto Nacional de la Juventud, 2015). En estudios anteriores el sexo anal era una práctica muy poco frecuente (Gysling, Benavente y Olavarría, 1997). En placer sexual también se evidencian transformaciones, ya que "las mujeres presentan el promedio más alto para el grupo más joven”. (Mella, Oyanedel, Vargas y de Ugarte, 2015, p. 293). Comparando encuestas de distintos años, Palma (2006) constata que se prolonga la vida sexual de las mujeres adultas respecto de generaciones anteriores y cambia su discurso sobre sexualidad, incluyendo referencias al placer sexual y a las relaciones en contextos de ocasionalidad. Investigaciones anteriores mostraban que, entre las entrevistadas, "hay varias que tienen una vida sexual insatisfactoria, en la cual no se logra el goce sexual, se logra sólo a veces, o hay un componente de dolor” (Gysling, Benavente y Olavarría, 1997, p. 31).

Los estudios de género muestran que, en las generaciones actuales, “aparecen cuestionamientos importantes respecto a qué significa ser hombre y ser mujer en una relación de pareja con otro diferente, en un tiempo en que los referentes tradicionales están en crisis” (Sharim y Rhim, 2017, p. 4). Las mujeres de hoy día parecen buscar espacios personales, más allá de la rutina doméstica, lo que se ha traducido en una mayor diversidad en los arreglos domésticos en las parejas (Saldaña, 2018). Así, aun cuando entre las mujeres persiste una tensión entre maternidad y vida profesional (Lizana, 2008) y una división del trabajo más o menos tradicional en las familias (PNUD, 2010)

"se observan algunas rupturas con estas tendencias, con hombres crecientemente integrándose y responsabilizándose por las tareas reproductivas. Este involucramiento incidiría en las configuraciones identitarias masculinas, que están siendo permeadas por las prácticas cotidianas de trabajo doméstico, cuidado y crianza de hijos e hijas, impactando las distintas formas en que se vive la paternidad" (Saldaña, 2018, p. 198-199)

En concordancia con ello, un estudio entre adolescentes mostró que, en este grupo, predominaría una "masculinidad semitradicional”, caracterizada por "la mezcla y tensión de aspectos de una masculinidad tradicional arraigada en costumbres machistas, con rasgos de una masculinidad emergente o alternativa, fundada en los principios de respeto y equidad entre hombres y mujeres”. (Matamala y Rodríguez, 2010, p. 77). Lo mismo 
reporta Valdés en las nuevas generaciones, que relevan aspectos subjetivos, emocionales y comunicativos que no estaban tan presentes en generaciones anteriores. "Las nuevas representaciones sobre la paternidad muestran rupturas intergeneracionales apareciendo atributos vinculados a la cercanía afectiva y la comunicación con los hijos frente al padre autoritario, violento, distante y lejano, pero sostén económico de la familia”. (Valdés, 2009, p. 402).

\section{Metodología}

Se decidió trabajar con teleseries por varios motivos; por un lado, teníamos un cierto recelo de trabajar con los métodos convencionales de entrevistas o encuestas, ya que, en el caso de las primeras, resulta muy difícil, como lo muestran los estudios revisados (Valdés, 2005; Valdés, 2007a, 2007b), trabajar con grupos socioeconómicamente diversos, mientras que, en las encuestas, a veces las personas pueden dar respuestas más políticamente correctas que reales, como las mediciones sobre felicidad, que puntúan sorprendentemente alto (World Happiness Report, 2019) o la escasa declaración de homosexualidad (Ministerio de Desarrollo Social, 2017). Las teleseries, en cambio, presentan características que despiertan el interés sociológico, ya que, según sus mismos guionistas y directores, éstas tratan deliberadamente de que las personas se identifiquen con los personajes, se vean reflejados/as en los protagonistas de la trama, acercando la representación a la cotidianidad del televidente. Según un estudio con telespectadores, la mayor parte de ellos "atribuyó a los personajes rasgos posibles de reconocer en cualquier 'persona normal, como la gente que uno puede ver en la calle'” (Amigo, Bravo y Osorio, 2014, p. 139). Esta identificación se logra mediante focus groups, donde prueban y definen el argumento y los personajes de la teleserie, incluso su nombre y su banda sonora (Santa Cruz, 2003). De este modo, la realidad ficcionada se nutre de la realidad experienciada, que, una vez en el aire, difunde imágenes y representaciones que pueden tener un efecto performativo en la opinión pública, instalando problemáticas controvertidas como temas de debate público (Vásquez, 2016) ${ }^{7}$. Siguiendo a autores como Martín Barbero (2002), creemos que existen relaciones de intercambio mutuo entre la familia representada en las teleseries y la familia de la vida cotidiana, por lo que el análisis de la ficción podría entregar interesantes antecedentes para comprender la realidad de las familias.

Nos planteamos realizar un estudio interpretativo, que se nutre de los principios metodológicos de la etnografía, la técnica del bricoleur de Levi-Strauss (1994) y la grounded theory (Charmaz y Mitchell, 2001). De la etnografía se tomaron varios de sus principios, dentro de los cuales podemos destacar el "ve y hazlo" de Hammersley y Atkinson ${ }^{8}$, y la discusión contemporánea sobre la necesidad de que la etnografía se adapte a los nuevos objetos de estudio (Mosquera, 2008), como podría ser, en nuestro caso, las teleseries. Al respecto, Soriano estima que:

"la aproximación de los investigadores a nuevos problemas del mundo social les ha obligado a adaptar los procedimientos etnográficos tradicionales. Ha sido el método lo que se ha puesto al servicio de los objetos de estudio y no al revés. Eso, que para algunos se puede ver como un signo de debilidad científico, es una cualidad de la versatilidad metodológica de la etnografía delante de un mundo social cambiante que se resiste a ser observado desde un cientificismo dogmático” (2011, p. 10).

Para el muestreo de teleseries construyó un listado con el total de programas entre 2005 y 2015 de las estaciones tradicionalmente las emitían ${ }^{9}$, el cual se analizó en función de dos criterios: básicos y de argumento. Los criterios básicos tenían que ver con la época representada en la teleserie (que correspondiese al tiempo en que se filmó) y que tuviera cierto nivel de audiencia ${ }^{10}$. Para los criterios de argumento se recurrió a páginas web de teleseries ${ }^{11}$, que contenían resúmenes de los argumentos, cuya fiabilidad podríamos discutir, pero, de todos modos, representaban una forma de analizar todas las teleseries según un mismo patrón. Al estudiar los argumentos, priorizamos las teleseries donde la familia apareciera como elemento central y que hubiera presencia de hijos/as. Cada aspecto se puntuó entre 1 y 5 puntos, seleccionándose las teleseries con mayor puntaje (Vidal, 2015). Así, la muestra quedó constituida por 5 teleseries: "Alguien te mira” (2007), "Papi Ricky" (2007), "Dama y obrero" (2012), "Socias" (2013) y "Matriarcas" (2015) ${ }^{12}$. Se visionaron entre los 12 y 15 primeros capítulos de cada teleserie. 
Las etapas del registro de la información fueron: (i) el visionado general, donde vimos los capítulos completos e hicimos anotaciones generales; (ii) las categorías y el registro sistemático: se adecuaron las categorías originalmente planteadas y se hizo una descripción de las escenas con mayor detalle; (iii) momento descriptivo/individual: se construyó un relato con las redes familiares y de parentesco visionadas en los capítulos, incluyendo referencias al género y a la sexualidad; (iv) momento interpretativo/grupal: se hizo un análisis transversal de los programas visionados en función de las categorías que emergieron de la etapa dos.

\section{Resultados}

Los tipos de familia mayormente representados en las teleseries fueron las reconstituidas y, en segundo lugar, las monoparentales, en consonancia parcial con los estudios realizados en el país, que, si bien recogen como tipología de familia a la monoparental, no ocurre lo mismo con la reconstituida (Ministerio de Desarrollo Social, 2011, 2015). En “Socias”, su protagonista, Inés Ventura, estuvo casada con Octavio Acuña, del que tiene una hija llamada Valentina; se casa en segundas nupcias con Ricardo Ossandón, que también tiene un hijo de un matrimonio anterior. Monserrat Álvarez se separa de Federico Ibáñez, con quien tiene un hijo de nombre Mateo, e inicia una relación con Cristóbal Pérez, un periodista unos 20 años menor que ella. En consonancia con los nuevos modelos familiares y el cambio en las identidades de género masculinas (Araujo y Martuccelli, 2012; Gómez y Jiménez, 2015), la pareja principal de "Papi Ricky” se encuentra separada, ya que los distanció el embarazo de Catalina, por lo que Alicia es criada por un motoquero padre soltero, sin participación alguna de la madre. Prontamente, Ricky entrará en relación con Colomba Chaparro, previa aprobación de su hija Alicia ${ }^{13}$, destacándose el cambio en la relación con los hijos y la transformación de una familia, que al principio era nuclear biparental, pasa a monoparental y termina como reconstituida al afianzarse la relación entre Ricky y Colomba. En la misma teleserie, Úrsula Flores se separa de Antonio Noriega, con quien tiene tres hijos, y entra en una relación con Greco Ovalle, un colega de su exmarido bastante menor que ella. En Alguien te mira, Julián García y Matilde Larraín, pese a estar separados, mantienen muy buena relación entre sí, sobre todo en temas de su hijo. Al cabo de un tiempo, Julián entra en una fogosa relación con Eva Zanetti, la policía encargada de resolver los asesinatos en serie que aterrorizan a las mujeres santiaguinas, mientras que Matilde se involucrará con Mauricio Ossa, un periodista que trabaja con Zanetti en el intrincado enigma policiaco. Así, en su representación televisiva, las parejas se ven afectadas por la circunscripción temporal de sus lazos afectivos, fenómeno opuesto al amor romántico, descrito por Bauman, Beck, Giddens e Illouz como hemos señalamos.

En "Dama y obrero" se observan principalmente familias monoparentales. A Margarita, madre de Julio Ulloa, el protagonista, no se le conoce pareja, no hay referencias a su existencia. Engracia Hurtado está separada hace mucho de su marido, ya que, en un loco arrebato de celos empujó a la amante de su esposo por las escaleras, provocando la ruptura de la pareja. Antes de involucrarse con Ignacia, Julio tenía una relación con Mireya Ledezma, que vive sola con su padre, quien tampoco tiene pareja. De la protagonista de "Matriarcas", Diana Nazer, sólo sabemos que es viuda y que tiene un hijo que crió en solitario. En la misma teleserie, aparece Matilde Valdés, que correspondería a una madre soltera por elección (Moncó, Jociles y Rivas, 2011), ya que es una mujer virgen, que fue inseminada artificialmente con los espermios del hijo de Diana, producto de lo cual han nacido 6 hijas. "Papi Ricky" aborda el embarazo adolescente a través del personaje de Macarena Garay, que sorprende a toda la familia con un avanzado estado de gravidez, que nadie del grupo familiar había advertido.

En "Socias”, presenciamos una radicalización de la discusión sobre familia y parentesco, que ya venía planteando la teoría sociológica (Konvalinka, 2010; Stone, 2007). Allí se muestra a dos maridos expulsados de su casa por motivos de infidelidades reiteradas, que se van a vivir juntos y conversan sobre ver un partido de fútbol el fin de semana. Pablo le comenta a Federico que no podrá ver el partido con él, ya que quedó de verlo con su hija, argumentando que lo hace porque "son familia". Un desconcertado Federico le pregunta qué son ellos entonces, discusión que culmina con el acuerdo de que verán el partido todos juntos, ya que todos ellos son familia, una gran familia, que estaría compuesta por Federico y Pablo, sus respectivos hijos/as, exesposas y las actuales parejas de sus ex. Así, en concordancia con la menor importancia de las familias nucleares tradicionales en el Chile actual (Olavarría, 2000), las familias representadas en las teleseries se distancian de estos modelos, situación distinta a lo que ocurría en los 80 , donde casi el único modelo era la familia nuclear biparental (Vidal, 2015). 
En términos de relaciones de género, se observa una importante horizontalidad en el trato que tienen las parejas, probablemente porque la mayor parte de las mujeres son profesionales independientes, que tienen una vida que no se circunscribe al ámbito del hogar. Las parejas separadas con hijos en común mantienen una relación muy saludable, que no se observa forzada sino cercana; tienen acordados los horarios de visita y mantienen un alto grado de comunicación respecto de los hijos/as, tal como describen Araujo y Martuccelli (2012) y Sharim y Rihm, (2017). Así ocurre con Inés Ventura y Octavio Acuña en “Socias”; con Julián García y Matilde Larraín en “Alguien te mira”, por nombrar algunos. En temas de sexualidad, casi todos los personajes mantienen relaciones sexuales, incluyendo adolescentes y personas de la tercera edad, en consonancia con los estudios, que muestran un adelanto en la entrada a la sexualidad (González y otros, 2007; Instituto Nacional de la Juventud, 2015) y una prolongación de la actividad sexual en la tercera edad (Herrera, 2003; Palma, 2006). En "Papi Ricky”, la adolescente Pascuala Chaparro tiene sexo casual con un desconocido, Valentín Carrasco, quien, en una inversión de los roles tradicionales de género, le confiesa que era virgen. Reforzando la inversión, Pascuala se molesta con la confesión y le dice que para ella fue sólo sexo, que no quiere saber nada de él, mostrando un cambio en los roles de género poco frecuente en tiempos anteriores (Gysling, Benavente y Olavarría, 1997). También hay relaciones intergeneracionales, como el romance entre Benjamín Morandé y Camila, Woods, hermana de su esposa Tatiana en “Alguien te mira”. La prolongación de la actividad sexual en personas mayores se incorpora en el romance de Julita, madre de un conservador Leonardo Garay, con Segundo Marcos, un empelado del condominio donde transcurre la acción en "Papi Ricky”, lo que agrega un grado importante de tensión en la familia.

Las relaciones sexuales muchas veces se distancian de la tradicional escena del amor romántico propia de épocas anteriores (Sharim, Silva, Rodó y Rivera, 1996; Gysling, Benavente y Olavarría, 1997), aproximándose a un modelo con evidente predominio del ello, tal como describe Giddens con la sexualidad plástica. En una escena de “Socias”, Dolores Montt y Pablo Ventura están tan excitados que no pueden esperar a llegar a la pieza para sacarse la ropa; caen al suelo mientras se desnudan, pero no por eso dejan de besarse ni tocarse el cuerpo de manera un tanto brusca. En otra ocasión tienen sexo en el baño público, evidenciando una diversificación de los lugares de realización de las prácticas sexuales, donde la excitación se combina con la adrenalina generada por el temor a ser sorprendidos. Lo mismo sucede con Julián García y Eva Zanetti en “Alguien te mira”, que tienen una candente relación sexual en un estacionamiento del restaurant donde fueron a comer. Por otro lado, si bien no se muestra explícitamente, tanto en "Socias” como en “Alguien te mira”, se da a entender que los personajes tienen prácticas sexuales que combinan penetración vaginal, oral y/o anal, situación muy distinta a la retratada en los estudios de fines de los 90, donde muy pocas parejas practicaban sexo anal (Gysling, Benavente y Olavarría, 1996). De este modo, las teleseries recogen los cambios que describen los estudios, como la precoz iniciación sexual, el cambio en la sexualidad femenina y la mantención de la actividad en las personas mayores, así como también la diversificación del repertorio sexual.

Además, emerge un tipo de mujer que vive su sexualidad de manera distinta a la tradicional, como Dolores Montt en "Socias”, para quien los hombres sólo sirven para satisfacerse sexualmente. Dolores no tiene problemas en ir sola a una discoteca, conocer un hombre, llevarlo a su departamento y luego despedirlo una vez concluido el encuentro sexual, estableciendo una clara escisión entre sexualidad y afectividad, que sería propia de la modernidad. El comportamiento de Dolores coincide con los estudios de Bernández (2012) y del Consejo Nacional de Televisión (2009), y también con las investigaciones sobre sexualidad, donde las relaciones en contextos de ocasionalidad constituye una de las diferencias entre mujeres de distintas generaciones (Palma, 2006; Instituto Nacional de la Juventud, 2015). Estudios anteriores mostraban que, si bien la virginidad había perdido importancia, no tener sexo con cualquiera y/o haber tenido relaciones sexuales con un solo hombre continuaba siendo importante para las mujeres (Valdés, Benavente y Gysling, 1999)

También aparecen las modernas tecnologías de la información, usadas con motivaciones diversas, pero todas ligadas a la familia y la sexualidad. En “Alguien te mira”, Mauricio Ossa graba de manera furtiva sus relaciones sexuales, sin el conocimiento ni la anuencia de sus contrapartes, probablemente para verlas posteriormente en solitario. En "Socias”, Monserrat Álvarez busca pareja en aplicaciones de teléfonos celulares, estilo Tinder, lo que se relaciona con las consideraciones de Da Silva (2006) y de Belli, López, Feliú y Juárez (2009) sobre el impacto de las tecnologías en el ámbito de la sexualidad, que tímidamente se visibilizaban a comienzos de siglo (Hernández, 2002). No obstante, su uso no se limita a la sexualidad, ya que incorpora la 
reproducción asistida, profundizándose en las eventuales relaciones de parentesco que podrían surgir a partir de su utilización. Tal es el caso de "Matriarcas", cuya historia versa en torno a Diana Nazer y su hijo Alexis, quien años atrás, de manera reservada, vendió espermios a un banco de semen, los que fueron utilizados para inseminar distintas mujeres. El hecho sale a la luz cuando una actividad familiar es interrumpida intempestivamente por una mujer con una niña del brazo, que asegura es hija de Alexis. Cuando Diana se entera, comienza a buscar a quienes considera "sus nietos/as", búsqueda que culmina con el descubrimiento de que han nacido 33 bebés de los espermios de su hijo. La teleserie plantea la pregunta de si es posible que una abuela pueda considerarse familia con sus nietos, pese a que su hijo (el padre) no tenga ningún interés en ello. Por otro lado, no todas las mujeres les dijeron a sus maridos que habían sido inseminadas, por lo que algunos creen ser los padres biológicos de los hijos/as. Al saberse la verdad, se producen interesantes debates en torno a la paternidad, instalando claramente el mensaje de que padre es el que cría, no el que pone los espermios. Así, la teleserie pone en discusión tanto las concepciones tradicionales de familia como de parentesco, tal como Konvalinka, (2010) y Parkin y Stone (2007) consideran ocurre en las sociedades contemporáneas.

\section{Discusión y conclusiones}

En términos generales, existe alto grado de coincidencia entre las representaciones de familia, género y sexualidad que difunden las teleseries analizadas con los planteamientos de la teoría sociológica y los resultados de los estudios realizados en el país. La excepción la constituyen las familias reconstituidas, principal familia representada en las teleseries, pero escasamente abordada por las investigaciones nacionales. Esta omisión ha sido advertida por organismos de Naciones Unidas, que señalan que aun cuando, en América Latina, la familia nuclear sigue siendo la prevalente, engloba realidades que pueden ser muy diferentes entre sí (Cerruti y Binstock, 2009). Lo mismo ocurre con las familias homoparentales, que, si bien no aparecieron en las teleseries analizadas, sí han sido tratadas en otras, como "Puertas adentro” (2003), “¿Dónde está Elisa?” (2009), “Separados” (2012) y "No abras la puerta” (2014), que visibiliza los problemas económicos de las parejas lésbicas para acceder a las modernas -y costosas- tecnologías de reproducción asistida. Los estudios tampoco incorporan a la familia homoparental como categoría de familia ${ }^{14}$.

Al invisibilizar las familias reconstituidas y homoparentales, los estudios caen en una forma de no reconocimiento, acto lesivo desde la perspectiva de Honneth (1997), y que también destaca Bourdieu (1996) al referirse a las familias reconstituidas como las familias sin nombre ${ }^{15}$. De persistir esta omisión, los estudios estarían contribuyendo a difundir sólo una parte de las diversas realidades familiares existentes en el país, lo que vendría a ser una realidad sesgada, distorsionada, incluso ideologizada en el sentido que propone Larraín (2009), ya que desconsidera los cambios que experimentan las familias en Chile, los que se hace necesario develar, pues no resultan del todo evidentes, al menos no desde la perspectiva de los estudios revisados en cuanto a tipologías de familia. Además, alguien podría pensar que se nos está haciendo parecer que somos más conservadores de lo que en realidad somos, situación que ya habría ocurrido en ápocas anteriores en el país (Parrini, 1997, Rajevic, 2000).

Por otro lado, el tipo de mujer que difunden las teleseries analizadas se condice con lo que Bernández (2012) ha llamado la mujer fálica y el Consejo Nacional de Televisión (2009) mujer amazona; mujeres independientes que asumen ideales, conductas y actitudes tradicionalmente asociados al hombre. Incluso se llega a cuestionar el mandato de la maternidad, como hace Antonia del Solar en "Socias", al informarle a su novio que ha decidido priorizar su carrera de modelo por sobre la de ser madre. A su vez, los modelos masculinos se alejan de la tradicional imagen del macho proveedor insensible, mostrando varones interesados en participar activamente en la crianza de los hijos y en mostrarse afectivos con ellos, lo que es refrendado por los estudios nacionales (Olavarría, 2001, Valdés, 2009; Araujo y Martuccelli, 2012 y Saldaña, 2018).

Usando la terminología de Giddens, estaríamos frente a "relaciones puras”, de igualdad sexual y emocional entre sus integrantes. La sexualidad mostrada en la teleserie se corresponde con lo que el mismo autor denomina "sexualidad plástica" y con los resultados de las investigaciones nacionales, que muestran un adelanto en el inicio de la actividad sexual de las mujeres, sobre todo al comparar por tramos de edad, ya que las más jóvenes se inician antes, declaran más parejas sexuales que sus predecesoras y mayor acceso al placer 
(ADIMARK, 2017; Fernández, et al., 2013). A comienzos del 2000, el tema del placer sexual femenino recién se instalaba en la discusión pública y, en ese momento, parecía circunscrito a ciertos grupos de la población, mujeres de mayor estrato socioeconómico (Vidal, 2002). Así lo muestra un estudio con mujeres de distinto estrato socioeconómico, donde, entre las del alto:

"La mayoría ha(bía) superado inhibiciones y ha(bía) logrado una sexualidad satisfactoria y plena.... Por su parte, entre las entrevistadas de sectores bajos, sólo una mujer manifiesta disfrutar parcialmente de las relaciones sexuales, mientras que todo el resto se encuentra insatisfecha” (Valdés, Benavente y Gysling, 1999, p. 91) $)^{16}$.

Los estudios realizados hoy indican que el acceso al placer femenino es un fenómeno mucho más generalizado (Mella, Oyanedel, Vargas y de Ugarte, 2015).

Por otro lado, la tendencia a abordar temas controvertidos pareciese ser una característica que ha acompañado a las teleseries desde sus inicios (Santa Cruz, 2003; Camponovo, 2012). Ya en 1982, Puga estimaba que "no podemos concluir que la imagen de familia que transmiten las telenovelas sea ni constructiva, valóricamente hablando, ni actualizada, frente a las características y desafíos que presenta esta institución hoy en nuestra sociedad” (Puga, 1982, p. 67). Desde una perspectiva radicalmente distinta, las investigaciones actuales sostienen que las teleseries "han incorporado de manera persistente y crítica, diversas materias al debate público, como la desigualdad, la diversidad sexual, la violencia intrafamiliar, la situación de los sin casa o de los pueblos originarios, el abuso infantil, entre muchos otros” (Amigo, Bravo y Osorio, p. 2014, p. 136).

Empero, los autores no creen que la teleserie produzca el debate social, sino que éste resultaría del contexto social, político y cultural del país, que permite sobrepasar los márgenes de los discursos mediáticos conservadores. Así, no es que la teleserie impulse una perspectiva progresista en el debate social, "sino que se adecua a las nuevas subjetividades que se van produciendo en la sociedad” (Amigo, Bravo y Osorio, p. 2014, p. 143). No obstante, esta consideración podría ser puesta en cuestión a partir del análisis que hace Vásquez (2016) sobre "Alguien te mira", donde concluye que la teleserie "se valió de su estructura melodramática para exponer un tema contingente que en el año 2007 recién comenzaba a hablarse abiertamente y a ocupar la agenda política: el machismo, la dominación patriarcal y la misoginia con resultado de femicidio” (Vásquez, 2016, p. 448). Lo mismo podemos decir de la teleserie "Matriarcas", ya que las inseminaciones artificiales y los eventuales lazos familiares o de parentesco que pudiesen surgir de allí no parece ser un tema que la sociedad chilena se encuentre debatiendo acaloradamente, pero que lo instala la teleserie. Tampoco se discute de las parejas lésbicas que carecen de medios económicos para acceder a técnicas de reproducción asistida, ni tampoco de la posibilidad de que dos amigos separados puedan constituir una familia. De este modo, tenemos dudas de si estos programas no pretenden explícitamente promover la discusión sobre temas controvertidos, propiciando de alguna manera la producción de cambios en este ámbito.

\section{Agradecimientos}

El autor agradece a los doctores Claudio Ramos Zincke y Jaime Barrientos Delgado de la Universidad Alberto Hurtado por sus valiosos comentarios.

\section{Notas}

\footnotetext{
${ }^{1}$ Por ejemplo, es posible que un/a menor acepte que deba respetar a su padre por el hecho de ser su padre, pero no es tan claro que deba tener la misma actitud con la pareja de la madre, que no es su padre biológico y, en ocasiones, puede que tampoco su padre social.

${ }^{2}$ Las familias reconstituidas son aquellas donde "al menos uno de los adultos tiene un hijo de una relación anterior” (Giddens, 2010, p. 394) Rivas sostiene que "son familias formadas por matrimonios o uniones de hecho de separados y divorciados con hijos procedentes de una relación o relaciones anteriores” (Rivas, 2012, p. 30)

${ }^{3}$ En las familias negociadas a plazo "las situaciones individuales independizadas entran en una alianza contradictoria con el fin de intercambiar las emociones de una manera reglada y hasta nuevo aviso” (Beck, 2010, p. 124).
} 


\begin{abstract}
${ }^{4}$ Para Arriagada (2005), los cambios fundamentales son la disminución del tamaño de la familia y el mayor espaciamiento entre los hijos.

${ }^{5}$ Roudinesco, por su parte, plantea que la familia es aquello que "une por un período de extensión relativa a dos individuos en busca de
\end{abstract} relaciones íntimas o expansión sexual” (2006, p. 20).

${ }^{6} \mathrm{Al}$ comparar por tramos de edad, quienes tienen más parejas sexuales son quienes tienen entre 15 y 19 años, con 19.5 parejas (Instituto Nacional de la Juventud, 2015, p. 99).

${ }^{7}$ En trabajos anteriores hemos desarrollado este argumento sobre la base de cuatro consideraciones: (i) el objetivo explícito de representar la cotidianeidad, (ii) la evolución de los temas de interés público, (iii) la identidad nacional, la modernización y la democracia y (iv) los temas emergentes (Vidal y Donoso, 2015).

${ }^{8}$ Después de revisar muchos estudios de teleseries, programas de televisión, incluso comics, no encontramos alguno que orientara para el análisis de la información. En este sentido, los autores fueron esclarecedores, había que hacerlo y ver como quedaba, ya que "toda investigación es una actividad práctica que requiere el ejercicio de un juicio en el contexto; no se trata de seguir simplemente unas reglas metodológicas” (Hammersley y Atkinson, 1994, p. 39).

${ }^{9}$ Este artículo es una revisita a mi tesis doctoral (Vidal, 2015), que comparaba teleseries actuales y de los 80, donde el levantamiento de información se realizó en 2013. No obstante, en este artículo, incorporamos una teleserie del 2015, ampliándose el período original, aunque con esta última no se siguió el mismo procedimiento de selección. Como en los 80, ni Chilevisión ni Mega producían teleseries, se mantuvo el criterio de excluirlas del análisis actual.

${ }^{10}$ Se obtuvieron los niveles de audiencia disponibles, se sumaron y se dividieron por el número de teleseries, obteniéndose una audiencia promedio, que excluían los programas con baja recepción por parte del público.

${ }^{11}$ www.telenovelaschilenas.cl http://teleserieschilenas.blogspot.com, http://fotech.cl/portal_fotech/teleseries/index.php, http:// es.wikipedia.org/wiki/Anexo:Telenovelas_de_Chile

${ }^{12}$ Pese a que se analizaron sólo esas 5 teleseries, también se incluyen, a modo de ejemplo, referencias a otras no consideradas.

${ }^{13}$ Lo mismo ocurre cuando, en Socias, Monserrat Álvarez le pregunta a su hijo su parecer con que se vaya a vivir con ellos Cristóbal.

${ }^{14}$ Si bien la encuesta Casen preguntó por la orientación sexual de las personas, no incorporó a las familias homoparentales en las categorías propuestas (Ministerio de Desarrollo Social, 2017)

${ }^{15}$ Un análisis de la diversidad sexual a partir de la sociología del reconocimiento se encuentra en Vidal, 2016.

${ }^{16}$ Un estudio en el mundo marítimo portuario de 2002 se concluye que "en la visión de las mujeres, lo más importante que el hombre tenga lo que quiere en el plano sexual para que él se sienta bien, en desmedro de lo que las mujeres mismas puedan sentir” (Vidal y Donoso, 2002, p. 28-29).

\title{
Bibliografía
}

Adimark (2017). Primera encuesta de sexualidad. Santiago, Chile: GFK Adimark. Recuperado de http:// www.adimark.cl/estudios/documentos/microestudio\%20sexualidad\%202017.pdf

Amigo, B., Bravo, M. y Osorio, F. (2014). Telenovela, recepción y debate social. Cuandernos.info, (35), 135-145 doi: 10.7764/cdi.35.654.

Araujo, K. y Martucceli, D. (2012). Desafíos comunes. Retrato de la sociedad chilena y sus individuos. Tomo II. Santiago, Chile: Lom Ediciones.

Arriagada, I. (2005). Transformaciones sociales y demográficas de las familias latinoamericanas. En: T. Valdés y $\mathrm{X}$. Valdés (eds.) Familia y vida privada. ¿Transformaciones, tensiones, resistencias y nuevos sentidos? (pp. 9-40). Santiago, Chile: FLACSO-Chile, CEDEM, UNFPA.

Bauman, Z. (2010). Modernidad líquida. Buenos Aires, Argentina: Fondo de Cultura Económica.

Beck, U. (2010). La sociedad del riesgo. Hacia una nueva modernidad. Barcelona, España: Paidós. 
Beck-Gernsheim, E. (2003). La reinvención de la familia. En búsqueda de nuevas formas de convivencia. Barcelona, España: Paidós.

Belli, S., López, C., Feliu, J. y Juárez, A. (2009). Educación y sexualidad en las nuevas tecnologías de la información y la comunicación: sexo y emociones delante de una pantalla plana. Transatlántica de educación, (6), 103-113. Recuperado de https://dialnet.unirioja.es/servlet/articulo?codigo=3080595

Bernández, A. (2012). Modelos de mujeres fálicas del postfeminismo mediático: Una aproximación a Millenium, Avatar y Los juegos del hambre. Anàlisi 47, 91-112. Recuperado de http://eprints.ucm.es/22899/1/ mujeres_falicas_postfeminismo_analisi.pdf

Bourdieu, P. (1996). Las familias sin nombre. Actes de la Recherche en Sciences Sociales 113. Recuperado de http://pierre-bourdieu.blogspot.com/2006/07/familias-sin-nombrepierre-bourdieu.html

Camponovo, S. (2012). La telenovela chilena: Radiografía de un cambio cultural, 1990-2012 (Tesis de Maestría). Universidad de Leiden, Países Bajos.

Cerrutti, M. y Binstock, G. (2009). Familias latinoamericanas en transformación: Desafíos y demandas para la acción pública. Serie Políticas Sociales (147), Santiago, Chile: CEPAL, UNFPA.

Consejo Nacional de Televisión (2009). Caracterización de los estereotipos de género en las telenovelas chilenas: el caso de Canal 13 y TVN. Santiago, Chile: Consejo Nacional de Televisión. Recuperado de http:// www.cntv.cl/prontus_cntv/site/artic/2 $0110317 /$ asocfile/20110317142005/ caracterizacion_estereotipos_genero_telenovelas_2009.pdf

Charmaz, K. y Mitchell, R. (2001). Grounded theory in etnography. En P. Atkinson, A. et al. (Eds.) Handbook of Etnography. (pp. 160-174). London, England: Sage Publications.

Da Silva, D. (2006). Deseos públicos e identidades privadas: Internet, género e identidad sexual masculina en Chile. El caso de los avisos personales del diario La Nación. Gazeta de Antropología (22). Recuperado de http://www.gazeta-antropologia.es/?p=2694

Del Picó Rubio, J. (2011). Evolución y actualidad en la concepción de familia. Una apreciación de la incidencia positivista de las tendencias dominantes a partir de la reforma del derecho matrimonial chileno. Revista Lus et Praxis, año 17, (1), 31-56.

Fernández, A., Celis-Atenas, K., Córdova-Rubio, N., Duffey, M., Correa, M. y Benedetti, J. (2013). Sexualidad juvenil: prácticas, actitudes y diferencias según sexo y variables de personalidad en universitarios chilenos. Revista médica de Chile, 141(2), 160-166. doi: http://dx.doi.org/10.4067/S0034-

Giddens, A. (2003). Un mundo desbocado. Los efectos de la globalización en nuestras vidas, Madrid, España: Taurus.

Giddens, A. (2008). La transformación de la intimidad. Amor, sexo y erotismo en las sociedades contemporáneas, Madrid, España: Cátedra.

Giddens, A. (2010). Sociología, Madrid, España: Alianza.

Gómez, V. y Jiménez, A. (2015). Corresponsabilidad familiar y el equilibrio trabajo familia: medios para mejorar a equidad de género. Polis, 14(40), 377-396. https://dx.doi.org/10.4067/S0718-65682015000100018

González, E., Molina, T., Montero, A., Martínez, V. y Leyton, C. (2006) Comportamientos sexuales y diferencias de género en adolescentes usuarios de un sistema público de salud universitario. Rev Méd Chile. 135: 12611269. Recuperado de: https://scielo.conicyt.cl/pdf/rmc/v135n10/art05.pdf 
Gonzálvez, H. (2016) Las familias transnacionales ¿una tautología? Más allá de la dicotomía distancia/proximidad geográfica. Polis, 15 (43), 511-532. Recuperado de https://scielo.conicyt.cl/pdf/polis/v15n43/art_24.pdf

Gysling, J., Benavente, M. y Olavarría, J. (1999). Sexualidad en jóvenes universitarios. Santiago, Chile: FLACSOChile.

Hammersley, M. y Atkinson, P. (1994). Etnografía. Métodos de investigación. Barcelona, España: Paidós.

Hernández, L. (2002). Sexo virtual. La escisión definitiva entre el estar y el placer. En F. Vidal y C. Donoso. Cuerpo y sexualidad (pp. 97-104). Santiago, Chile: FLACSO-Chile, Universidad ARCIS, VIVO POSITIVO.

Herrera, A. (2003). Sexualidad en la vejez: ¿mito o realidad? Revista chilena de obstetricia y ginecología, 68(2), 150-162. https://dx.doi.org/10.4067/S0717-75262003000200011

Herrera, F. y Teitelboim, B. (2010). La mirada de los chilenos a la familia. En Universidad Diego Portales. Encuesta Nacional UDP (pp. 121-135). Santiago, Chile: Universidad Diego Portales.

Honneth, A. (1997). La lucha por el reconocimiento. Por una gramática moral de los conflictos sociales, Barcelona, España: Crítica.

Illouz, E. (2009). El consumo de la utopía. El amor y las contradicciones culturales del capitalismo, Buenos Aires, Argentina: Katz editores.

Instituto de Estudios de la Sociedad (2014). A propósito de Nicolás y sus papás. Santiago, Chile. Recuperado de http://www.ieschile.cl/2014/11/a-proposito-de-nicolas-y-sus-papas/

Instituto Nacional de la Juventud (2012). VII Encuesta Nacional de Juventud, Santiago, Chile: Ministerio de Desarrollo Social

Instituto Nacional de la Juventud (2012). VIII Encuesta Nacional de Juventud, Santiago, Chile: Ministerio de Desarrollo Social

Konvalinka, N. (Ed.) (2012). Modos y maneras de hacer familia. Las familias tardías, una modalidad emergente. Madrid, España: Siglo XXI.

Larraín, J. (2007) El concepto de ideología. Tomo I. Santiago, Chile: Lom Ediciones.

Levi-Strauss, C. (2014). Pensamiento salvaje, Ciudad de México, México: Fondo de Cultura Económica.

Lizana, V. (2008). Representaciones sociales sobre feminidad de los/las estudiantes de pedagogía, en los contextos de formación docente inicial. Estudios pedagógicos, 34 (2), 115-136. https://dx.doi.org/10.4067/S071807052008000200007

Martín-Barbero, J. (2002). La telenovela desde el reconocimiento y la anacronía. En H. Herlinghaus (Ed.), Narraciones anacrónicas de la modernidad. Melodrama e intermedialidad en América Latina. (pp. 62- 77). Santiago, Chile: Editorial Cuarto Propio.

Matamala, M. y Rodríguez, M. (2010). Estudio exploratorio sobre la identidad de género de hombres adolescentes pertenecientes al sector barrio norte de concepción. Última década 18 (33), 61-84. Recuperado de: https:// scielo.conicyt.cl/scielo.php?script=sci_arttext\&pid=S0718-22362010000200005

Mella, C., Oyanedel, J., Vargas, S. y de Ugarte, N. (2015). Salud sexual en Chile: Una aproximación descriptiva al comportamiento y la satisfacción sexual de los chilenos, Revista chilena de obstetricia y ginecología, 80 (4), 289-296. doi: http://dx.doi.org/10.4067/S0717-75262015000400003. 
Ministerio de Desarrollo Social (2015). Panorama CASEN 2015. Hogares y bienestar: Análisis de cambios en la estructura de los hogares (1990-2015), Santiago, Chile. Recuperado de: http:// observatorio.ministeriodesarrollosocial.gob.cl/documentos/Panorama_Casen_2015_Hogares_y_bienestar.pdf

Ministerio de Desarrollo Social (2017) Casen 2017. Equidad de Género. Santiago, Chile. Recuperado de: http:// observatorio.ministeriodesarrollosocial.gob.cl/casen-multidimensional/casen/docs/ CASEN_2017_EQUIDAD_DE_GENERO.pdf

Ministerio de Planificación (2009). Encuesta de caracterización socioeconómica (CASEN). Santiago, Chile. Recuperado de http://observatorio.ministeriodesarrollosocial.gob.cl/layout/doc/casen/casen_2009_familia.pdf

Ministerio de Planificación (2011). Mujer y familia. CASEN 2011. Santiago, Chile. Recuperado de http:// centrodelafamilia.uc.cl/Descargar-documento/1462-Resultados-Mujer-y-Familia-CASEN-2011.html

Ministerio de Salud (2000). Estudio nacional de comportamiento sexual. Santiago, Chile: Comisión Nacional del SIDA

Moncó, B., Jociles, M. y Rivas, A. (2011). Madres solteras por elección: representaciones sociales y modelos de legitimación. Nueva Antropología. 24 (74), 73-92. Recuperado de http://www.scielo.org.mx/ scielo.php?pid=S0185-06362011000100004\&script=sci_arttext

Morandé, P. (1994). Persona, matrimonio y familia. Santiago, Chile: Ediciones de la Universidad Católica de Chile.

Mosquera, M. (2008). De la etnografía antropológica a la etnografía virtual. Estudio de las relaciones sociales mediadas por internet. Revista Venezolana de Sociología y Antropología 18 (53), 532-549. Recuperado de: http://www.redalyc.org/pdf/705/70517572006.pdf

Olavarría, J. (2000). De la identidad a la política: Masculinidades y políticas públicas. Auge y ocaso de la familia nuclear patriarcal en el siglo XX. En J. Olavarría, y R. Parrini. (Eds.). Masculinidades, identidad, sexualidad y familia. Pp. 11-28. Santiago, Chile: FLACSO-Chile.

Olavarría, J. (2001). Y todos querían ser (buenos) padres. Santiago, Chile: FLACSO-Chile.

Olavarría, J. (2014). Transformaciones de la familia conyugal en Chile en el período de la transición a la democracia (1990-2011). Polis, 13, (37), 473-497. doi: http://dx.doi.org/10.4067/S0718-65682014000100025

Organización de Estados Americanos. (2008). Derechos humanos, orientación sexual e identidad de género. Washington. Recuperado de http://www.oas.org/es/sla/ddi/ derechos_humanos_orientacion_sexual_identidad_genero.asp

Palma, I. (2006). Sociedad chilena en cambio, sexualidades en transformación (Tesis de doctorado). Universidad de Chile. Santiago, Chile.

Parkin, R. y Stone, L. (eds.) (2007). Antropología del parentesco y de la familia. Madrid, España: Editorial Universitaria Ramón Areces.

Parrini, V. (1997). Matar al minotauro. Chile. ¿Crisis moral o moral en crisis? Santiago, Chile: Planeta.

PNUD (2012). Desarrollo Humano en Chile. Bienestar subjetivo: el desafío de repensar el desarrollo. Santiago, Chile: PNUD.

Puga, J. (1982). Las telenovelas: Valores y anti-valores. Santiago, Chile: Centro Bellarmino, Departamento de investigaciones sociológicas. 
Rajevic, P. (2000). El libro abierto del amor y el sexo en Chile. Santiago, Chile: Planeta.

Registro Civil (2016). Primer boletín de información semestral 2016 servicio de Registro Civil e Identificación Ministerio de Justicia y Derechos Humanos. Santiago, recuperado de http://www.registrocivil.cl/PortalOI/ PDF/Boletin_Semestral_SRCeI_2016_Datos.pdf

Rodríguez, C. y Martínez, K. (2012). El contrato de maternidad subrogada: La experiencia estadounidense. Revista de derecho 25(2), 59-81. https://dx.doi.org/10.4067/S0718-09502012000200003

Rodrigo, M. y Palacios, J. (Coords) (1998). Familia y desarrollo humano. Madrid, España: Alianza.

Roudinesco, E. (2006). La familia en desorden. Ciudad de México, México: Fondo de Cultura Económica.

Rivas, A. (2012). El ejercicio de la parentalidad en las familias reconstituidas. Portularia, XII (2), 29-41. doi 10.5218/prts.2012.0042

Saldaña, L. (2018) Relaciones de género ya arreglos domésticos: Masculinidades cambiantes en Concepción, Chile. Polis, 17, (50). 183-204. http://dx.doi.org/10.4067/S0718-65682018000200183.

Santa Cruz, E. (2003). Las telenovelas puertas adentro. El discurso social de la telenovela chilena. Santiago, Chile: LOM.

Sharim, D., Silva, U., Rodo, A. D. Rivera. (1996). Los discursos contradictorios de la sexualidad. Santiago, Chile: SUR.

Sharim, D. y Rihm, A. (2017) Desigualdades de género: el conflicto en las relaciones de intimidad. Centro de Estudios de Conflicto y Cohesión Social. Recuperado de: https://www.coes.cl/wp-content/uploads/2017/10/ NCPP07.-Desigualdades-de-genero.pdf

Soriano, J. (2011). Las nuevas reglas de la etnografía de la comunicación. Portal de la Comunicación. Institut de la comunicació, Universitat Autónoma de Barcelona. Recuperado de http://www.portalcomunicacion.com/ lecciones_det.asp?id=30

Stone, L. (2007). ¿Ha girado el mundo? Parentesco y familia en la telenovela americana contemporánea. En R. Parkin y L. Stone. Antropología del parentesco y de la familia (pp. 647-666). Madrid: Editorial Universitaria Ramón Areces.

Universidad Católica y ADIMARK (2006). Encuesta Nacional Bicentenario. Centro de Políticas Públicas. Santiago, Chile. Recuperado de http://www.emol.com/noticias/documentos/pdfs/encuesta_bicentenario21 1006.jpg2.pdf

Universidad Católica y ADIMARK (2016). Encuesta Nacional Bicentenario. Centro de Políticas Públicas, Santiago, Chile. Recuperado de http://encuestabicentenario.uc.cl/wp-content/uploads/2016/11/Encuesta-bicentenario-2016-Familia.pdf

Valdés, T., Benavente, M. y Gysling, J. (1999) El Poder en la Pareja, la Sexualidad y la Reproducción. Mujeres de Santiago. Santiago: FLACSO-Chile.

Valdés, T. (2005). ¿Del deber al placer? Socialización en sexualidad en familias populares en Santiago. En T. Valdés y X. Valdés (Eds.) Familia y vida privada. Transformaciones, tensiones, resistencias y nuevos sentidos (pp. 311-337). Santiago, Chile: FLACSO-Chile, CEDEM.

Valdés, X. (2007a). Notas sobre la metamorfosis de la familia en Chile. Santiago, Chile: CEPAL, UNFPA. 
Valdés, X. (2007b). Vida en común. Familia y vida privada en Chile y el medio rural en la segunda mitad del siglo $X X$. Santiago, Chile: LOM Ediciones.

Valdés, X. (2009). El lugar que habita el padre en el Chile contemporáneo. Estudio de las representaciones sobre la paternidad en distintos grupos sociales. Polis, 8, (23), 385-410. Recuperado de https://scielo.conicyt.cl/ scielo.php?script=sci_abstract\&pid=S0718-65682009000200017\&lng=es\&nrm=iso

Vásquez, A. (2016). Femicidio en la telenovela Alguien te mira. Metáfora de un país misógino. Polis, 15 (44), 435456. doi: http://dx.doi.org/10.4067/S0718-65682016000200020.

Vidal, F. (2002) Sexualidad y Modernidad en Chile: Una Relación Espúrea”. En F. Vidal y C. Donoso. Cuerpo y sexualidad (pp. 27-44). Santiago, Chile: FLACSO-Chile, Universidad ARCIS, VIVO POSITIVO.

Vidal, F. y Donoso, C. (2002). Estrategias de Empoderamiento y Prevención del VIH/SIDA en Parejas de Trabajadores Marítimo Portuarios. Santiago: FLACSO-Chile, Universidad ARCIS, VIVO POSITIVO.

Vidal, F. y Donoso, C. (2015). Los nuevos modelos de género y sexualidad presentes en las teleseries chilenas. $V$ Coloquio Internacional de estudios sobre varones y masculinidades, Santiago. Recuperado de: https:// mega.co.nz/\#F!IhZAwDhT!NqwiIFCcYBlsi_Tw94ckqg

Vidal, F. (2015). La familia representada en las teleseries chilenas: Un análisis comparativo de programas de los años 80 y post 2000 (Tesis de doctorado). Universidad Alberto Hurtado, Santiago, Chile.

Vidal, F. (2016): Sociología del reconocimiento y diversidad sexual: Desafíos para la inclusión educativa. Diálogos Educativos, XVI, (31) 151-176. Recuperado de http://www.dialogoseducativos.cl/revistas/n31/vidal.pdf 